\title{
Intelligent decision support system based geo-information technology and spatial planning for sustainable water management in Flanders, Belgium
}

\author{
H.A. Saleh \\ Sustainable Mobility, Ghent University, Belgium \\ G. Allaert \& R. De Sutter \\ Institute for Sustainable Mobility, Ghent University, Belgium \\ W. Kellens \& Ph. De Maeyer \\ Department of Geography, Ghent University, Belgium \\ W. Vanneuville \\ Flanders Hydraulics Research, Authorities of Flanders, Antwerpen, Belgium
}

Development Centre, Ministry of Local Administration and Environment, Damascus, Syria Institute for

ABSTRACT: The paper outlines the main features of an intelligent decision support system based on existing and planned tools for optimising water management and flood risk reduction. Up to now, flood risk is increasing and environmental degradation is continuing; this requires developing robotic algorithms that can provide a degree of functionality for spatial representation and flexibility suitable for creating real-time solutions that maximize the urban flood protection measures. Moreover, the volume of data collected is growing rapidly and sophisticated means to efficiently optimise the data are essential. There is a need to develop a shared information system for flood management which will promote model and systems integration, monitoring, and decision making in strategic planning and emergency situations. This advanced area of research is a promising direction for producing an effective time-efficient solution to flood risk reduction where other methods failed. Therefore, the objective of this paper is to bring together innovative methods in the field of artificial intelligence, geoinformation technology and spatial and environmental planning to achieve more effective water management and flood risk reduction in Flanders.

Keywords: Artificial intelligence; decision support; flood management; sustainable spatial planning

\section{INTRODUCTION}

Floods are regularly recurring natural disasters caused by extreme weather conditions, and in comparison with other natural disasters, floods can often cause tremendous economic damage and lead to environmental emergency situations that compromise the integrity of large infrastructures and the lives of many human beings. This is clearly evident in the recent devastation caused by Hurricane Katrina in 2006, when historic flooding affected not only the New Orleans area, but three coastal states in the USA. Over the last decade, Europe has experienced a number of unusually long-lasting rainfall events that resulted in severe floods - in the Netherlands, France and Germany $(1993,1995)$; the Czech Republic, Poland and Germany (1997); North Italy (1994, 2000); the UK $(1998,2000)$; and recently in Germany, Austria, the Czech Republic, Slovalia, Russia, and Romania $(2002,2004)$. At the same time, Flanders has suffered repeated major flooding events (December 1993, January 1995, September 1998, and December 1999), and wide areas of the region were inundated causing significant damage to many essential infrastructures, as well as distress to the local population. Based on the increasing frequency and magnitude of flood events and responding to the above critical situation, the Flemish water administrations (Flanders Hydraulics Research (FHR) and Flemish Environmental Agency Water Department) encouraged several programmes for its flood management policy and to establish a preventive approach of flood protection. FHR has already developed several computer models of the most important streams to imitate the floods and to predict their geographical extent. This is done in online and offline mode. The offline study mode is used for scenario 
calculations based on synthetic hydrographs while the online mode uses more than 400 rainfall, water level, velocity and discharge measuring stations sending values to the database with intervals between one minute and 60 minutes. To calibrate these models, an inundation database is provided with the models and contains the natural flooding areas (NOG) and the recently flooded areas (ROG) in Flanders from 1988 to 2005 (NOG/ROG data base). The database has been built up with information from local authorities, Flemish administrations and consultancy agencies. Also, the database is used as an important instrument for the policy of regional planning and the operational water system management. Statutory maps for the Watertoets (water test) or disaster insurance law are based on a combination of modelling results and the ROG database.

The research described here will effectively support an existing programme called 'LATIS' which has been developed by the Department of the Geography, Ghent University, for flood management at Flanders Hydraulics Research. The main objective is the development of a new and improved methodology to optimise the functionality of the existing model, using robotic and innovative procedures, based on the ideas of artificial intelligence. The developed methodology will take into account all relevant aspects of flood management: preventive measures, water management, land-use, urban development at all levels (local, regional, and national), monitoring and forecasting overflows, early warning, simulation and optimisation procedures, etc. This will generate knowledge contributing to the design of effective response actions that maximise the urban flood protection and safety measures.

\section{THE CURRENT SITUATION OF FHR-RISK MODEL PROGRAMME}

Because of the complexity of flood prevention, the research has concentrated on achieving a better understanding of causes, methods of prediction and management of floods and their damage, and preventative risk reduction by sustainable spatial planning (e.g., landuse control, regional and urban development planning, etc.). The paper will describe the planned improvement and optimisation stages of FHR-Risk Model programme which is in use at FHR and many engineering companies for studies ordered by public and private organisations in Belgium and Europe. It is used for the EU InterregIIIb projects, COMRisk and SAFECoast, and is one of the base methods for the social cost benefit analysis of the New Sigma Plan (Schelde Area) and the Integrated Coastal Safety Plan (a description of the modelling tools used and some results can be found in (Vanneuville et al 2003, 2005) and (Verwaest et al 2005)). Compared to previous approaches where only water levels and discharges were taken into account, this methodology allows a more objective comparison between different hydrographic catchments. Instead of working with freeboards based on known water levels, the FHR-Risk Model is based on robust time series statistics and Peak Over Threshold (POT) selection for the derivation of return periods. However, the model still has several weak points on important technical issues such as: working with a very fine grid (making the calculation times very long), no optimal use due to the doubles (calculations are sometimes done twice in different sub-modules of the program), not enough output information about levels of uncertainty around the result, and limited knowledge about the propagation of input errors in the result, etc. Some of the uncertainty questions are solved partly based on case studies and parameter variation, but due to calculation times and model restrictions an additional system is needed.

The developed system will be designed to consider several levels of planning and decision making through the management of spatially referenced data with advanced computer simulation, graphical visualisation, and dynamic metaheuristc methods. Several important elements must be integrated into a new strategy that will be practically embedded in the current model. The proposed strategy involves aspects of: (1) spatial planning and land-use regulation (e.g., declaration of flood risk areas as priority and reserve areas, etc), (2) water management (e.g., determination of flood areas, installation of flood action plans, and installation of regional flood concepts, etc.), and (3) risk management (e.g., flood forecasting, implementation of early-warning systems, and development of flood hazard and vulnerability maps, etc.).

\section{RULES OF SPATIAL PLANNING IN FLOOD MANAGEMET}

As a consequence of the enormous economic damage caused by recent floods, various flood mitigation measures -and not only structural measures such as dams and dikes- must be combined in an integrated approach to flood management (Friesecke, 2004). Flood reduction is part of sustainable urban water development and there are methods of improving preventive flood protection based on spatial planning and urban development:

1) Protection of existing retention areas: e.g., declaration of flood areas, determining the access to rivers, cleaning out the riverbeds, protecting and increasing the vegetation areas on the banks, the execution of a water test for all future interventions in the river bed, the banks, retention areas, and keep controlling the disposal within environmental permission, etc. 
2) Extension of retention areas: e.g., backward relocation of dikes, creating detention ponds, restoration of large streams, flood plain scrapes/deepening of retention areas, etc.

3) Retention in the catchments: e.g., rainwater storage and grey water use, restriction of sealed surface, reduction of interflow on agricultural and forestry land, restoration of small streams, etc.

4) Minimisation of damage potential: e.g., preventive land-use management, precautionary measures of construction, information of the public, improvement of public awareness, prediction of floods and warning, disaster prevention/control, etc.

5) Technical flood protection measures: e.g., dikes, flood protection walls, retention ponds, river dams, barrages, etc.

Flood risk can only be effectively reduced if, in addition to technical measures, spatial planning strategies are used to regulate land-use in flood-prone areas. Precautionary and sustainable spatial planning efforts must strive to achieve a balance between economic urban development and urbanisation, while securing more space for water retention. Land-use planning plays a vital role in preventive flood management and directly contributes to flood control (determination of flood risk areas), and indirectly contributes to the minimisation of flood hazards (reforestation, avoidance of sealing). In addition, flood mapping is an important basis for all flood damage reduction programmes.

\section{INTELLIGENT DECISION SUPPORT SYSTEM (IDSS)}

It is envisioned that the main component of the conceptual framework for flood management is a decision support system (Loucks, et al 1991). The main innovative aspect of the developed system is the integration of a comprehensive geographical and environmental data collection, and data management tools with simulation and decision tools for flood management. Metaheuristic algorithms (which are based on the ideas of artificial intelligence) potentially have the ability to produce sets of high quality real-time designs that can model more closely and easily many objective functions, visualize the trade-offs between them, and then filter and cluster top optimal solutions (Osman and Kelly, 1992). In addition, metaheuristics can provide instantaneous comparisons of the achieved results of different developed designs using several procedures such as convergence, diversity, and complexity analysis. This will allow the modeller to develop a precise and unambiguous specification that can help in estimating the impacts of the proposed metaheuristics on an actual development process in the presented design. Currently, it is almost impossible even for

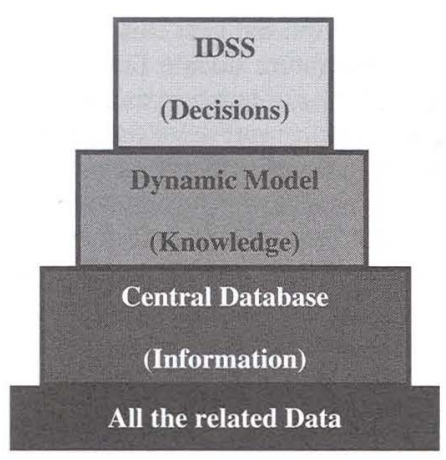

Figure 1. Structure of the Intelligent Decision Support System (IDSS).

an experienced designer to find an optimal design using current methods, as they do not provide spatial representation of the whole situation and lack the ability to select 'interesting' contingencies for which to optimise. Once such designs are obtained, the technical-user will be able to select an acceptable design by trading off the competing objectives against each other, as well as taking into account other practical considerations. The final design should be robust (i.e., performs well over a wide range of environment conditions), sustainable (i.e., not only optimal under current conditions, but also taking into account predicted changes in climate and hydrology parameters), and flexible (i.e., allows easy adaptation after the environment has changed).

\subsection{Metaheuristic techniques}

The developed system will be combined with more classical techniques of engineering analysis, data processing and computer simulation and coupled with metaheuristic techniques. The well-known optimisation metaheuristics that have been successfully applied to real-life applications are: simulated annealing, tabu search, ant colony optimization, and genetic algorithm (Saleh and Dare, 2002). These metaheuristics are inspired, respectively, by the physical annealing process, the proper use of memory structures, the observation of real ant colonies, and the Darwinian evolutionary process.

\subsubsection{Simulated Annealing (SA) technique}

The SA technique is flexible, robust and capable of producing the best solution for complex real-life problems (Aarts and Van Laarhoven, 1995). This technique is derived from physical science and is based on a randomisation mechanism that creates solutions and accepts the best one. The annealing parameters that have to be specified are: initial temperature, the temperature update function, the length of the Markov chain and the stopping criterion. The initial temperature simulates the effect of temperature in the 
search process to find the best candidate of the final design. The temperature update function determines the behaviour of the cooling process, while the length of the Markov chain represents the number of iterations between successive decreases in temperature. The optimisation process is terminated at a temperature low enough to ensure that no further improvement can be expected. With suitable annealing parameters, an optimal (or close to optimal) solution to a flooding problem can be achieved for (Saleh and Dare, 2001).

\subsubsection{Tabu Search (TS) technique}

The TS technique, which is a global iterative optimisation, exploits knowledge of the system or "memory" under investigation to find better ways to save computational efforts without effecting solution quality (Glover and Laguna, 1997). The most basic form of the TS is the construction of a tabu list which prevents the search from cycling by forbidding certain candidates and then directing the search towards the global optima. At the beginning of the process, this list is often empty but is created during the search process by the addition of candidates that could return the current candidate to previous local optima. Implementation of TS requires specification of tabu parameters: the tabu list, the candidate list, the tabu tenure, and the stopping criteria. The tabu list is a memory structure that prohibits moves that have recently been interchanged to prevent cycling from occurring. The candidate list contains a set of selected moves that gives the bestgenerated neighbouring candidates that surround the current candidate. The tabu tenure determines the number of iterations for which a candidate maintains its tabu status (Saleh and Dare, 2003).

\subsubsection{Ant Colony Optimization (ACO)}

The ACO is a multi-agent approach to search and reinforce solutions in order to find the optimal ones for hard optimization problems. This technique is a biological-inspired agent based on the foraging behaviour of real ant colonies for distributed problemssolving (Dorigo and Gambardella, 1996). The basic idea underlying this metaheuristic is the use of chemical cues called pheromone (form of collective memory). The function of this pheromone is to provide a sophisticated communication system between ants that cooperate in a mathematical space where they are allowed to search and reinforce pathways (solutions) in order to find the optimal one. This metaheuristic includes positive feedback (intensity to quickly discover good solution), distributed computation (to avoid premature convergence), and the use of a constructive greedy metaheuristic (visibility to help find an acceptable solution in the early stage of the search process) (Saleh, 2002a).

\subsubsection{Genetic Algorithms (GAs)}

Unlike the above mentioned techniques, GAs, which are inspired from population genetics, operate on a finite pool of solutions (usually called chromosomes) (Goldberg, 1989). The chromosomes are fixed strings with binary values at each position. The main idea behind GAs is to maintain this pool of selected solutions that evolves under selective pressure that favours better solutions. To facilitate production of better solutions and prevent trapping in local optima, a set of genetic operators are used. These operators include cross-over, mutation, and inversion. In cross-over, some cut-points (members of the population) are chosen randomly and the information between these chromosomes is exchanged. The mutation operator prevents GAs from trapping in local optima by selecting a random position and changing its value. In Inversion, two-cut points are chosen at random and the order of the bits is reversed (Saleh and Chelouah, 2003).

\subsection{Geo-information technology}

More than $85 \%$ of all the information used by water management is geographically referenced or, at least, geo-coded. The developed system utilizes the strengths of geo-information technologies (Geographic Information Systems (GIS), Remote Sensing (RS), Global Navigation Satellite Systems (GNSS), Internet, etc) in providing and representing spatial data, and dynamic models in analysing and representing temporal processes. Satellite imagery, which is in digital format, allows for the acquisition of environmental data and land occupation patterns and features over large areas. The main limitations of satellite images are cloud cover and resolution. Some of these problems may be circumvented using GNSS receivers. When associated with GIS, a GNSS receiver is the main reliable source for quick and accurate on-line information as well as a powerful dynamic mapping tool (Leick, 1995). Vegetation, land-use patterns, surface waters, quality and humidity of the soil, tracking the environmental characteristics and changes useful to the study of freshwater resources; changes in climate may be monitored by GNSS satellites. GIS facilitate the integration of quantitative water determination and control data with data obtained from maps, aerial photos, satellite images and satellite navigation systems (Saleh, 2002b).

\subsection{The Objectives of IDSS and its central database}

The developed decision support system will be connected to a power database to effectively optimise flood management over other existing methods by:

1) Providing access through a multiple-level webbased interface to a wide range of data types collected at investigated region in real-time. The user interface includes a module for computer 
simulation of different flood scenarios, a tool for managing simulation results, communication tools, etc. This, for example, will help to optimise the stream gages locations and their operations for flood predictions (including early warning and cost-benefit analysis).

2) Combining the observational data with innovative data analysis to improve forecasting and risk assessment and analysing and providing a clear physical representation of the processes involved that define risk zones and emergency scenarios (which is one of the main limitations in the existing model) (Saleh and Allaert, 2005). For example, values of water depth, velocity and their combination, and the flood time are visualised in a global map, providing a useful tool for emergency management and for determining protective measures against floods. This will support a human interface and allow the technical-user to interact with the current representation of the design, enhance the user's understandings, make it quicker for information to be reached on time, and react properly to warnings.

3) Developing advanced computational methods for collecting, processing, and generating the data necessary for the fast and accurate simulation of different flood situations and the $3 \mathrm{D}$ visualization of numerical results. This will: a) give the synthesise results of monitoring data from different sources, models, data analysis, etc; b) support the evaluation of the effect of alternative response scenarios by optimising the information overload (i.e., how to filter information and still get the right information, to the right people, at the right time); and c) assist in establishing the social, economic and environmental goals for managing floods.

4) Developing a flood warning network for assisting in real-time emergency services (Saleh, 2006). Metaheuristics can successfully handle a mix of continuous and discrete parameters, as well as select individual components from the database. The network will be connected to a database that combines environmental and geophysical data from earth observation, satellite positioning systems, in-situ sensors and geo-referenced information with advanced computer simulation and graphical visualisation methods (Peng, et al 2002). The database will provide the following internet-based services: quickly locate and ensure data availability where and when needed; detailed descriptions of contents and limitations of the data; and present the data in different formats (maps, graphs, pictures, videos, etc.). In addition, the database will be designed to be searchable by data type, data holder/owner, location, etc, and will be used in three modes: planning and design for flood protection; real-time flood emergency; and flood recovery.

\section{CONCLUSION}

Flood protection is becoming more and more important in meeting sustainable water development objectives. In order to be effectively prepared for floods, interdisciplinary and precautionary measures with regard to water management, spatial planning, and land management are necessary to increase protection measures and reduce flood damage. The proposed research constitutes a crucial step in water management by elucidating how artificial intelligence and spatial planning could be efficiently introduced in the design process of flood prevention to create dynamic optimisation methods that potentially reduce damage. In comparison to the use of metaheuristic methods to optimise other real-life applications in the domain of disaster management and risk reduction, these methods can potentially provide vital information that is quicker, better, and at a lower cost than existing methods (Saleh and Dare, 2000). The project will also show how a novel approach to the parallelisation and hybridisation of metaheuristics, coupled with local search procedures, can simplify the handling of data, minimize execution time, and facilitate the design modelling approach based on the simulation and optimisation process (April et al 2003). Furthermore, a sensitivity analysis, using an anticipatory process, will be performed in order to handle robustness and simulate an appropriate behaviour of the design parameters in real-time (Glover et al 2004). This project, by developing a new methodology for effectively optimising the use of these technologies, coupled with a longterm sustainable spatial planning strategy, can help to increase protection measures and reduce flood damage in Flanders.

\section{REFERENCES}

April, J., Glover, F., Kelly, J. and Laguna, M. (2003). Practical Introduction to Simulation Optimisation, Proceedings of the 2003 Winter Simulation Conference, New Orleans, USA.

Arts, E. and Van Laarhoven, P. (1985). Statistical Cooling: A General Approach to Combinatorial Problems. Phillips Journal of Research, 40, 213-225.

Deb, K., (2001). Multi-objective Optimisation using Evolutionary Algorithms. Wily \& Sons, Ltd, Chichester.

Dorigo, M. and Gambardella, L. M., (1996). Ant Colony System: a Cooperative Learning Approach to the Traveling Salesman Problem. IEEE Transactions on Systems, Man and Cybernetics-Part B, 26, 29-41.

Friesecke, F. (2004). Precautionary and Sustainable Flood Protection in Germany-Strategies and Instruments of Spatial Planning, Proceedings of the 3rd FIG Regional Conference, Jakarta, Indonesia.

Glover, F. and Laguna, M. (1997). Tabu Search, Kluwer Academic Publishers, Boston, USA.

Glover, F., Cavin, L. and Fischer, U. (2004). Multi-objective process design in multi-purposes batch plants using Tabu 
Search optimisation algorithm. Computers and Chemical Engineering, 28(4), 459-478.

Goldberg, D. E. (1989). Genetic Algorithms in Search, Optimization and Machine Learning. Addison-Wessley Publishing Company, Inc, USA.

Loucks, D.P., and daCosta, J.R. (eds.). (1991). Decision Support Systems: Water Resources Planning. SpringerVerlag, Berlin.

Osman, I. and Kelly, J. (1992). Meta-heuristic: An overview. In: Meta-heuristics: Theory and Applications, Dordrecht: Kluwer Academic Publishers.

Peng, G., Leslie, L. and Shao, Y. (2002). Environmental Modelling and Prediction. Springer-Verlag, New York.

Saleh, H. and Dare, P. (2000). Local search strategy to produce schedules for a GPS surveying network, Tutorial \& keynote papers of the 11th YOR Conference, Cambridge, UK, 28-30 March.

Saleh, H. and Dare, P. (2001). Effective Heuristics for the GPS Survey Network of Malta: Simulated Annealing and Tabu Search Techniques. Journal of Heuristics. 7(6), 533-549.

Saleh, H. (2002a). Ants can successfully design GPS Surveying Networks. GPS World, 13(9), 48-60.

Saleh, H. (2002b). Metaheuristics for optimising the use of Geographic Information Systems, Proceedings of the Euro-conference of the Science for Water Policy (SWAP): The Implications of the Water Framework Directive, East Anglia. UK.

Saleh, H. and Dare, P. (2002). Heuristics for Improved Efficiency in the Use of the Global Navigation Satellite
Systems (GNSS) for Establishing Positioning Networks. Marie Curie Fellowship Annals (MCFA), II, 62-74.

Saleh, H. and Dare, P. (2003). Near-optimal design of Global Positioning System Networks using Tabu Search Technique. Journal of Global Optimization, 25, 183-208.

Saleh, H. and Chelouah, R. (2003). The design of the Global Navigation Satellite Surveying Networks using Genetic Algorithms. Journal of the Engineering Applications of Artificial Intelligence, 17(1), 111-122.

Saleh, H., and Allaert, G. (2005). Dynamic Optimization for Environmental Pollution Control and Risk Management, Proceedings of the Sustainable development for the Syrian Coast, Lattakia, Syria.

Saleh, H. (2006). Space and Information Technologies for Designing Disaster Warning Network. Proceeding of the 15th International Symposium on Remote Sensing and Assisting Systems, Damascus, Syria.

Vanneuville, W., De Maeyer, Ph., Maeghe, K., and Mostaert, F. (2003). Model the effects of a flood in the Dender catchement based on a risk methodology, Society of Cartography Bulletin, 37 (2), 59-64.

Vanneuville, W., De Rouck, K., Deschamps, M., De Maeyer, Ph., and Mostaert, F. (2005). Spatial Calculation of Flood Damage and Risk Ranking. Proceedings of AGILE2005, Lissabon, Portugal.

Verwaest, T., and Trouw, K. (2005). Risk Assessment into Flanders-COMRisk Subproject 6. Die Küste, 70, $75-85$. 